\title{
New Error Coefficients for Estimating Quadrature Errors for Analytic Functions
}

\author{
By Philip Rabinowitz and Nira Richter
}

Abstract. Since properly normalized Chebyshev polynomials of the first kind $\widetilde{T}_{n}(z)$ satisfy

$$
\left(\tilde{T}_{m}, \tilde{T}_{n}\right)=\int_{\epsilon \rho} \tilde{T}_{m}(z) \overline{T_{n}(z)}\left|1-z^{2}\right|^{-1 / 2}|d z|=\delta_{m n}
$$

for ellipses $\epsilon_{\rho}$ with foci at \pm 1 , any function analytic in $\epsilon_{\rho}$ has an expansion, $f(z)=\sum a_{n} \widetilde{T}_{n}(z)$ with $a_{n}=\left(f, \widetilde{T}_{n}\right)$. Applying the integration error operator $E$ yields $E(f)=\sum a_{n} E\left(\tilde{T}_{n}\right)$. Applying the Cauchy-Schwarz inequality to $E(f)$ leads to the inequality

$$
|E(f)|^{2} \leqq \sum\left|a_{n}\right|^{2} \sum\left|E\left(\tilde{T}_{n}\right)\right|^{2}=\|f\|^{2}|| E \|^{2} .
$$

$\|E\|$ can be computed for any integration rule and approximated quite accurately for Gaussian integration rules. The bound for $|E(f)|$ using this norm is compared to that using a previously studied norm based on Chebyshev polynomials of the second kind and is shown to be superior in practical situations. Other results involving the latter norm are carried over to the new norm.

1. Davis and Rabinowitz [6], following the work of Davis [3], developed a new method for bounding the truncation error in the numerical integration of functions analytic over the interval of integration, standardized to $[-1,1]$. This method was based on the fact that every such function could be continued analytically into a region enclosed by one of a family of confocal ellipses $\epsilon_{\rho}$, with foci at \pm 1 , where $\rho=a+b, a$ is the semimajor axis of $\epsilon_{\rho}$, and $b=\left(a^{2}-1\right)^{1 / 2}$ is the semiminor axis. Error coefficients $\sigma(R, \rho)$ were computed for various values of $\rho$ and for several integration rules $R$, where

$$
R(f)=\sum_{i=1}^{n} w_{i} f\left(x_{i}\right)
$$

is determined by a particular choice of weights $w_{i}$ and abscissas $x_{i}, i=1, \cdots, n$.

The $\sigma(R, f)$ were computed using the Chebyshev polynomials of the second kind $U_{n}(z)$ which are orthogonal over the interior of $\epsilon_{\rho}$ with respect to the inner product

$$
(f, g)_{\rho}^{\prime}=\iint_{\epsilon_{\rho}} f(z) \overline{g(z)} d x d y .
$$

They are given explicitly by $t^{\prime}$-e formula

$$
\sigma^{2}(R, \rho)=\frac{4}{\pi} \sum_{k=0}^{\infty} \frac{(k+1)\left[\frac{1+(-1)^{k}}{k+1}-\sum_{i=1}^{n} w_{i} U_{k}\left(x_{i}\right)\right]^{2}}{\rho^{2 k+2}-\rho^{-2 k-2}} .
$$

Received November 26, 1968, revised December 18, 1969.

AMS Subject Classifications. Primary 6555, 6580.

Key Words and Phrases. Error coefficients, error in numerical integration, analytic functions, Chebyshev polynomials, complete orthonormal set, error estimates, trapezoidal rule, Simpson rule, norm of error functional, interpolatory quadrature.

Copyright @ 1971, American Mathematical Society 
The error $E(f)$ in the numerical integration of a particular function $f(x)$

$$
E(f)=\int_{-1}^{1} f(x) d x-R(f)
$$

can then be bounded

$$
|E(f)| \leqq \sigma(R, \rho)\|f\|_{\rho}^{\prime},
$$

where $\|f\|_{\rho}^{\prime 2}=(f, f)_{\rho}^{\prime}$. $\rho$ can take on any value $1<\rho<\bar{\rho}$, where $\bar{\rho}$ is the largest value of $\rho$ such that $f(x)$ is analytic in $\epsilon_{\rho}$. There is usually a value of $\rho, \rho^{*}$, such that

$$
\sigma\left(R, \rho^{*}\right)\|f\|_{\rho}^{\prime} \leqq \sigma(R, \rho)\|f\|_{\rho}^{\prime},
$$

for all $\rho, 1<\rho<\bar{\rho}$, so that

$$
|E(f)| \leqq \sigma\left(R, \rho^{*}\right)\|f\|_{\rho}^{\prime} .
$$

In practice, ||$f \|_{\rho}^{\prime}$ is usually estimated by $(\pi a b)^{1 / 2} M_{\rho}(f)$, where $M_{\rho}(f)=\max _{z \in \epsilon_{e}}|f(z)|$ and $\pi a b$ equals the area of the interior of $\epsilon_{p}$. Hence, the bound on $E(f)$ takes the form

$$
|E(f)| \leqq(\pi a b)^{1 / 2} \sigma(R, \rho) M_{\rho}(f) .
$$

In the present work, we introduce new error coefficients $\tau(R, \rho)$ using the Chebyshev polynomials of the first kind $T_{n}(z)$, which are orthogonal on $\epsilon_{\rho}$ with respect to the inner product

$$
(f, g)=\int_{\epsilon_{p}} \frac{f(z) \overline{g(z)}}{\left|1-z^{2}\right|^{1 / 2}}|d z| .
$$

These coefficients will turn out to give sharper bounds on $|E(f)|$ when used in the form similar to (6). In fact, for an integration rule whose error term starts with $C f^{(n)}(\xi)$, use of $\tau(R, \rho)$ will give error bounds which are asymptotically of magnitude $(n+1)^{-1 / 2}$ times the error bound (6) as $\rho$ tends to infinity.

Since the original work involving the $\sigma(R, \rho)$ led to further developments by Davis [4], Hämmerlin [7], Barnhill and Wixom [1], and others, we developed corresponding results for the $\tau(R, \rho)$. Following the example of Stroud and Secrest [11], we computed $\tau(R, \rho)$ for various Gaussian integration rules and selected values of $\rho$. However, we do not give a table of values but, instead, give a simple expression which, in almost all cases, overestimates $\tau$ and with an error of less than $10 \%$. We do give a table of coefficients useful for estimating the error in using the composite trapezoidal and Simpson rules, similar to that given by Hämmerlin [7]. Finally, we discuss some new rules which minimize $\tau(R, \rho)$ similar to what Barnhill and Wixom did with respect to $\sigma(R, \rho)$.

2. Let $L^{2}\left(\epsilon_{\rho}\right)$ denote the class of functions $f(z)$ which are single-valued and analytic inside $\epsilon_{\rho}$, such that

$$
|| f \|_{\rho}^{2}=\int_{\text {, }}|f(z)|^{2}\left|1-z^{2}\right|^{-1 / 2}|d z|
$$

is finite. For any two functions in $L^{2}\left(\epsilon_{\rho}\right)$, we can define an inner product $(f, g)_{p}$ by (7). The polynomials 


$$
p_{n}(z)=\left(\frac{2}{\pi}\right)^{1 / 2}\left(\rho^{2 n}+\rho^{-2 n}\right)^{-1 / 2} T_{n}(z)
$$

form a complete orthonormal set with respect to (7) $\left[5\right.$, p. 240]. Here, $T_{n}(z)=$ $\cos [n \operatorname{arc} \cos z], n=0,1,2, \cdots$, are the Chebyshev polynomials of the first kind. Every function $f(z)$ in $L^{2}\left(\epsilon_{\rho}\right)$ can be expanded in a Fourier series

$$
f(z)=\sum_{n=0}^{\infty} a_{n} p_{n}(z)
$$

where $a_{n}=\left(f, p_{n}\right)$, and the convergence is uniform and absolute in any closed subset of $\epsilon_{\rho}$. Moreover, we have

$$
\|f\|_{\rho}^{2}=\sum_{n=0}^{\infty}\left|a_{n}\right|^{2} .
$$

Let $\bar{E}$ be a bounded linear functional over $L^{2}\left(\epsilon_{\rho}\right)$. Then

$$
\|E\|_{\rho}^{2}=\sum_{n=0}^{\infty}\left|E\left(p_{n}\right)\right|^{2}
$$

and. for all $f \in L^{2}\left(\epsilon_{p}\right)$,

$$
|E(f)| \leqq\|\bar{E}\|_{\rho}\|f\|_{\rho} .
$$

In the present case, the functional of interest is the error in numerical integration

$$
E(f)=\int_{-1}^{1} f(x) d x-\sum_{i=1}^{n} w_{i} f\left(x_{i}\right)=I(f)-R(f) .
$$

In this case, we write $\tau(R, \rho)=\|E\|_{\rho}$ and, in view of (9) and (12), we have

$$
\tau^{2}(R, \rho)=\frac{2}{\pi} \sum_{k=0}^{\infty}\left(\rho^{2 k}+\rho^{-2 k}\right)^{-1}\left|E\left(T_{k}\right)\right|^{2} .
$$

Since $I\left(T_{k}\right)=2\left(1-k^{2}\right)^{-1}$ for $k$ even and $I\left(T_{k}\right)=0$ for $k$ odd, we can compute $\tau^{2}$ in terms of the weights and abscissas of the rule $R$ as

$$
\tau^{2}(R, \rho)=\frac{2}{\pi} \sum_{k=0}^{\infty}\left(\rho^{2 k}+\rho^{-2 k}\right)^{-1}\left[I\left(T_{k}\right)-\sum_{i=1}^{n} w_{i} T_{k}\left(x_{i}\right)\right]^{2} .
$$

If $R(f)$ is an integration rule which is exact for polynomials of degree $<m$, this reduces to

$$
\tau^{2}(R, \rho)=\frac{2}{\pi} \sum_{k=m}^{\infty}\left(\rho^{2 k}+\rho^{-2 k}\right)^{-1}\left[\frac{1+(-1)^{k}}{1-k^{2}}-\sum_{i=1}^{n} w_{i} T_{k}\left(x_{i}\right)\right]^{2} .
$$

If, furthermore, $R(f)$ is a symmetric rule exact for polynomials of degree $<2 m$, we have

$$
\tau^{2}(R, \rho)=\frac{2}{\pi} \sum_{k=m}^{\infty}\left(\rho^{4 k}+\rho^{-4 k}\right)^{-1}\left[\frac{2}{1-4 k^{2}}-\sum_{i=1}^{n} w_{i} T_{2 k}\left(x_{i}\right)\right]^{2} .
$$

Using formula (18), we computed values of $\tau\left(G_{n}, \rho\right)$ for values of $n$ and $\rho$ similar to those given by Stroud and Secrest [11] and, in Fig. 1, we give a graph for the $\tau\left(G_{n}, \rho\right)$ similar to the graph in [11] for the $\sigma\left(G_{n}, \rho\right)$, where $G_{n}$ denotes the $n$-point Gauss 


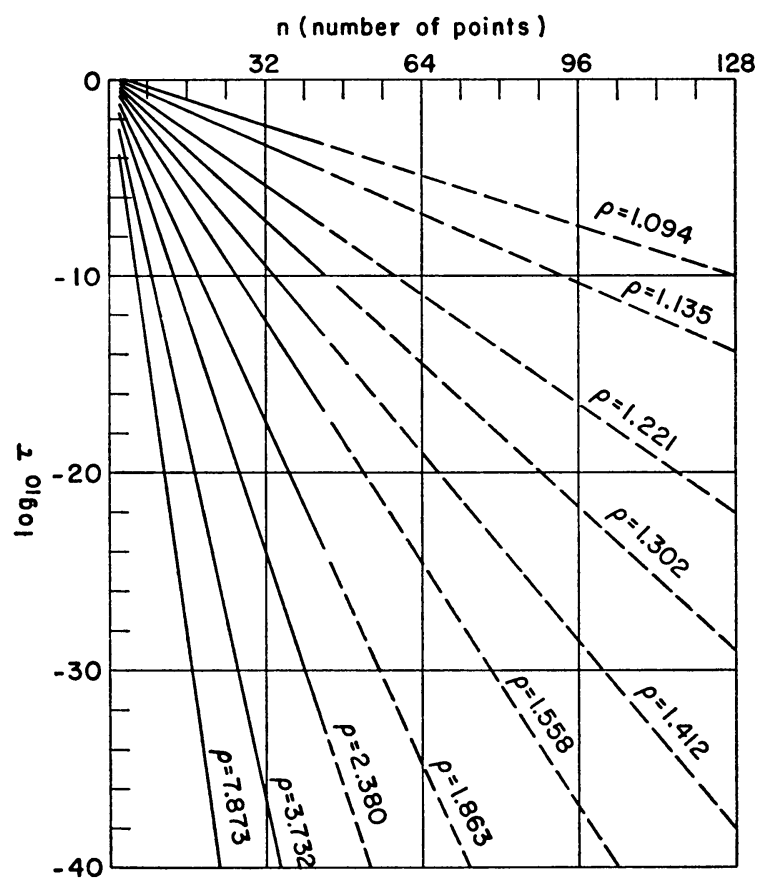

Fig. $1 \tau\left(G_{n}, \rho\right)$ as a function of $\rho$ and $n$.

colculated.

- extrapolated

integration rule. However, we do not list the values computed and, instead, give the following expression as an estimate for $\tau\left(G_{n}, \rho\right)$ :

$$
\tau\left(G_{n}, \rho\right) \sim\left(\frac{\pi}{2}\right)^{1 / 2} \frac{1}{\rho^{2 n}}\left[1+\frac{1}{2 \rho^{4}}\left(1+\frac{1}{2 n}\right)^{2}\right] .
$$

This estimate, which we shall justify immediately, turned out to overestimate $\tau$ for all pairs $(n, \rho)$, in the table in [11] except for $\rho=1.0935$ and $n=2,3$ where the true values of $\tau$ were 1.400 and 1.084 and the estimates gave 1.355 and 1.081 , respectively. (Note that we use here $\rho=a+b$ whereas in [11] $\rho=(a+b)^{2}$.) The maximum amount by which it overestimated $\tau\left(G_{n}, \rho\right)$ was by $10 \%$. For small $\rho$, the error was about $5 \%$, while for $\rho>1.8$ and $n>20$, the error was less than $1 \%$.

This estimate is based on the work of Nicholson et al. [8] who showed that

$$
E_{\theta_{n}}\left(T_{2 n+2}\right)=-C_{n}\left[1+\frac{(2 n+1)}{(2 n-1)(2 n+3)}\right],
$$

where

$$
C_{n} \equiv E_{G_{n}}\left(T_{2 n}\right)=\frac{2 \cdot 2 \cdot 4 \cdot 4 \cdots(2 n) \cdot(2 n)}{1 \cdot 3 \cdot 3 \cdot 5 \cdots(2 n-1) \cdot(2 n+1)}<\frac{\pi}{2}
$$

and that $\lim _{n \rightarrow \infty} E_{G_{n}}\left(T_{2 n+2 k}\right)=0$ for all $k>1$. Hence, we can estimate $\tau^{2}\left(G_{n}, \rho\right)$ by the first two terms in (18), 


$$
\begin{aligned}
\tau^{2}\left(G_{n}, \rho\right) & \sim \frac{2}{\pi} C_{n}^{2}\left[\frac{1}{\rho^{4 n}+\rho^{-4 n}}+\frac{\left[1+\frac{(2 n+1)}{(2 n-1)(2 n+3)}\right]^{2}}{\rho^{4 n+4}+\rho^{-4 n-4}}\right] \\
& <\frac{\pi}{2} \frac{1}{\rho^{4 n}}\left[1+\frac{(1+1 / 2 n)^{2}}{\rho^{4}}\right] .
\end{aligned}
$$

Therefore

$$
\begin{aligned}
\tau\left(G_{n}, \rho\right) & \sim\left(\frac{\pi}{2}\right)^{1 / 2} \frac{1}{\rho^{2 n}}\left[1+\frac{(1+1 / 2 n)^{2}}{\rho^{4}}\right]^{1 / 2} \\
& <\left(\frac{\pi}{2}\right)^{1 / 2} \frac{1}{\rho^{2 n}}\left[1+\frac{(1+1 / 2 n)^{2}}{2 \rho^{4}}\right],
\end{aligned}
$$

which is our desired estimate. Here, we used the elementary inequality $(1+x)^{1 / 2}<$ $1+x / 2$ for $x>0$. An alternative inequality $\left(1+x^{2}\right)^{1 / 2}<1+x$ leads to the simpler expression

$$
\left(\frac{\pi}{2}\right)^{1 / 2} \frac{1}{\rho^{2 n}}\left[1+\frac{1}{\rho^{2} 2}\left(1+\frac{1}{2 n}\right)\right],
$$

but this is not as accurate as the one chosen. We should point out that the estimate is for the theoretical value of $\tau$. For practical use, $\tau(R, \rho)$ should generally be computed using (15). See Rabinowitz [9].

In using (13) to estimate the error, we must have a way to compute $\|f\|_{\rho}$. There are several ways of estimating $\|f\|_{\rho}$ which are similar to the methods for estimating the norm of $f$ with respect to (2) given in [6]. The most accessible method is to write

$$
\|f\|_{\rho}^{2} \leqq \max _{s \in \epsilon_{\rho}}|f|^{2} \int_{e_{\rho}} \frac{|d z|}{\left|1-z^{2}\right|}=2 \pi M_{\rho}(f)^{2},
$$

so that

$$
\|f\|_{\rho} \leqq(2 \pi)^{1 / 2} M_{\rho}(f) .
$$

We now have two estimates for $|E(f)|$ in terms of $M_{\rho}(f)$, one of the form

$$
|E(f)| \leqq(\pi a b)^{1 / 2} \sigma(R, \rho) M_{\rho}(f)
$$

and the other, of the form

$$
|E(f)| \leqq(2 \pi)^{1 / 2} \tau(R, \rho) M_{\rho}(f) .
$$

We now state the following result:

THEOREM. Let $R(f)$ be an integration rule which has an error term of the form $E(f)=C f^{(n)}(\xi)+$ terms involving derivatives of order $>n, C \neq 0,-1 \leqq \xi \leqq 1$. Then the ratio of the right-hand side of (22) to that of (21) tends to $(1 /(n+1))^{1 / 2}$ as $\rho$ tends to infinity, i.e.,

$$
\lim _{\rho \rightarrow \infty}\left(\frac{2}{a b}\right)^{1 / 2} \frac{\tau(R, \rho)}{\sigma(R, \rho)}=\left(\frac{1}{n+1}\right)^{1 / 2} .
$$

Proof. Consider the expression

$$
r(R, \rho)=\frac{2}{a b} \frac{\tau^{2}(R, \rho)}{\sigma^{2}(R, \rho)},
$$


for large $\rho$. Since $a=\frac{1}{2}\left(\rho+\rho^{-1}\right), b=\frac{1}{2}\left(\rho-\rho^{-1}\right)$, we have $a b \sim \frac{1}{4} \rho^{2}$. Furthermore,

$$
\tau^{2}(R, \rho) \sim \frac{2}{\pi} \frac{\left|E\left(T_{n}\right)\right|^{2}}{\rho^{2 n}}
$$

and

$$
\sigma^{2}(R, \rho) \sim \frac{4}{\pi}(n+1) \frac{\left|E\left(U_{n}\right)\right|^{2}}{\rho^{2 n+2}}
$$

Hence,

$$
r(R, \rho) \sim \frac{4\left|E\left(T_{n}\right)\right|^{2}}{(n+1)\left|E\left(U_{n}\right)\right|^{2}} .
$$

Now. since $E(f)=C f^{(n)}(\xi)+\cdots$, we have

$$
E\left(T_{n}\right)=C 2^{n-1} n ! \text { and } E\left(U_{n}\right)=C 2^{n} n ! .
$$

Hence, $r(R, \rho) \sim 1 /(n+1)$, which proves the theorem.

Values of $r^{1 / 2}\left(G_{n}, \rho\right)$ were computed for different values of $n$ and show that the convergence to $(1 /(2 n+1))^{1 / 2}$ is quite rapid. The above theorem was proven for the special case of Gauss-Legendre quadrature by Chawla [2].

3. In this section we shall follow Hämmerlin [7] and compute new error coefficients $\tau^{*}(T, \rho)$ and $\tau^{*}(S, \rho)$ for the composite trapezoidal and Simpson rules, respectively. Although it is possible to compute $\tau(R, \rho)$ for any particular trapezoidal or Simpson rule containing $n$ subintervals, nevertheless it is impractical to tabulate such values in view of the range of $n$. Hence, it is desirable to have error coefficients independent of $n$, which, when multiplied by a suitable function of $n$, depending on the rule, give values of use in bounding the integration error.

In the trapezoidal case, the error estimate takes the form

$$
|E(f)| \leqq h^{2} \tau^{*}(T, \rho)\|f\|_{\rho},
$$

while in the Simpson case it is

$$
|E(f)| \leqq h^{4} \tau^{*}(S, \rho)\|f\|_{\rho},
$$

where $h=2 / n$, the length of the subinterval chosen. We shall sketch the computation of $\tau^{*}(T, \rho)$ since all the details are parallel to those given in [7] for the corresponding case. The considerations for $\tau^{*}(S, \rho)$ are similar and need not be given.

In the trapezoidal case, we have by the Euler-Maclaurin formula that

$$
E\left(T_{2 k}\right)=-\alpha_{2, k} h^{2}+\alpha_{4, k} h^{4}-\cdots,
$$

where

$$
\alpha_{2 i, k}=\frac{B_{2 i}}{(2 j) !}\left[T_{2 k}^{(2 i-1)}(1)+T_{2 k}^{(2 i-1)}(-1)\right]
$$

and

$$
B_{2 i}=\frac{2(-1)^{i-1}(2 j) !}{(2 \pi)^{2 i}} \sum_{n=1}^{\infty} n^{-2 i}
$$


are the Bernoulli numbers. We can show that for $1 \leqq k \leqq K$ and for any $h$,

$$
\left|E\left(T_{: 2:}\right)\right| \leqq \alpha_{:, l:} h^{2} \text {, }
$$

so that, by (15),

$$
\frac{\pi}{2} \tau(T, \rho)^{2} \leqq \sum_{k=1}^{k} \frac{\left(\alpha_{2, k} h^{2}\right)^{2}}{\rho^{4 k}+\rho^{-4 k}}+\sum_{k=K+1}^{\infty} \frac{\left|E\left(T_{2 k}\right)\right|^{2}}{\rho^{4 k}+\rho^{-4 k}} \equiv \Sigma_{1}+\dot{\nu}_{2} .
$$

Choosing $K$ so that $\Sigma_{2} / \Sigma_{1}<10^{-4}$, we then have to 3 -figure accuracy

$$
\tau(T, \rho) \leqq\left(\frac{2}{\pi} h^{+} \sum_{k=1}^{K} \frac{\alpha_{2, k}^{2}}{\rho^{4 k}+\rho^{-i k}}\right)^{1 / 2}=h^{2} \tau^{*}(T, \rho) .
$$

where

$$
\tau^{*}(T, \rho)=\left(\frac{2}{\pi} \sum_{k=1}^{K} \frac{\alpha_{2, k}^{2}}{\rho^{4 k}+\rho^{-4 k}}\right)^{1 / 2}
$$

Similarly, we have

$$
\tau^{*}(S, \rho)=\left(\frac{2}{\pi} \sum_{k=2}^{K_{1}} \frac{\beta_{2, k}^{2}}{\rho^{4 k}+\rho^{-4 k}}\right)^{1,2}
$$

where

$$
\beta_{2, k}=\frac{2^{4}}{3 \cdot 4 !}\left(1-\frac{1}{2^{2}}\right) B_{4}\left(T_{2 k}^{3)}(1)-T_{2 k}^{(3)}(-1)\right)
$$

is the coefficient of $h^{4}$ in the error expansion

$$
E_{s}\left(T_{2 k}\right)=\beta_{2, k} h^{4}+\beta_{3, k} h^{6}+\cdots \text {. }
$$

To show that (28) holds, we consider three ranges. In the range $1 \leqq n \leqq n_{1}(k)$, we have

$$
\left|E\left(T_{2 k}\right)\right|=\left|\frac{-1}{4 k^{2}-1}-h \sum_{j=0}^{n} \prime^{\prime \prime} T_{2 k}(-1+h j)\right| \leqq \frac{1}{4 k^{2}-1}+2 \leqq \alpha_{2, k} h^{2} .
$$

For $n_{1}(k)<n \leqq n_{2}(k)$, we computed $E\left(T_{2 k}\right)$ exactly and verified that (28) holds for $k \leqq K$. For $n_{2}(k)<n<\infty$, we can prove that $\alpha_{4, k} h^{4} \leqq 2 \alpha_{2, k} h^{2}$ and that $\alpha_{2 r+2, k} h^{2 r+2} \leqq$ $\alpha_{2 r, k} h^{2 r}$, for $r \geqq 2$, which again shows that (28) holds. The proof follows that given by Hämmerlin for the case of $U_{2 n}$. Thus, for the particular values of $k, 1 \leqq k \leqq K$, for which we computed $E\left(T_{2 k}\right)$, we know that (28) holds, which is all that we need. The values $\tau^{*}(T, \rho)$ and $\tau^{*}(S, \rho)$ are given in Table 1 for various values of $\rho$.

4. For each integration rule $R$ and each value of $\rho,(15)$ defines the value of the norm of the error functional $E(f)$. The question then arises to find particular rules which minimize the value of $\tau$. This has been done by Barnhill and Wixom [1] with respect to the norm $\sigma$ for two different cases, and we do the same for $\tau$. In the first case, the abscissas are held fixed and the weights are chosen which minimize $\tau$. In the second case, both the abscissae and the weights of an $n$-point rule are allowed to vary so as to minimize $\tau$. In both cases, it is possible to impose constraints on the rules such as the constraint that the rules integrate constants exactly, i.e., $\sum_{i=1}^{n} w_{i}=2$. 
We computed unconstrained minimum norm rules for $n=2,3,4$ and various values of $\rho$ and similar rules for fixed abscissae. We also computed corresponding rules subject to the constraint that $\sum w_{i}=2$. These rules are essentially of no practical interest so that we shall not list them here. From the theoretical point of view, their asymptotic properties are of interest and these are discussed in [10]. For the present, we remark that the values of the minimum norm $\left\|R_{n}\right\|$ are about half those of $\tau\left(G_{n}, \rho\right)$ for small values of $\rho$ while, for large values of $\rho$, they are almost equal, and the abscissae and weights of the minimum norm rules approach those of the corresponding Gaussian rule. It is an easy matter to prove that, for any $n$, the limit as $\rho$ tends to infinity of any minimum norm rule is the $n$-point Gaussian rule. The proof is based on the fact that for large $\rho$,

$$
\tau^{2}(R, \rho) \sim \frac{2}{\pi} \frac{\left|E\left(T_{k}\right)\right|^{2}}{\rho^{2 k}},
$$

where $k$ is the first integer such that $E\left(T_{n}\right) \neq 0$. For the $n$-point Gaussian rule, $k=2 n$ while for any other rule, $k<2 n$. Hence, any rule which minimizes $\tau$ must tend to $G_{n}$.

5. In this section we shall extend some results of Davis [4] on interpolatory quadrature to the space $L^{2}\left(\epsilon_{p}\right)$. Consider a scheme of quadratures of the form

$$
\int_{1}^{1} f(x) d x \sim \sum_{k=0}^{n} w_{n k} f\left(x_{n k}\right)=I_{n}(f), \quad n=1,2, \cdots .
$$

It is said to converge uniformly in $L^{\prime 2}(B)$ if, given $\epsilon>0$, there exists $n_{0}=n_{0}(\epsilon)$ such that for all $f \in L^{\prime 2}(B)$ and $n \geqq n_{0},\left|E_{n}(f)\right|=\left|\int_{-1}^{1} f(x) d x-I_{n}(f)\right| \leqq \epsilon|| f||$. Here $L^{\prime 2}(B)$ is the set of functions analytic within the region $B$ containing $[-1,1]$ such that $\iint_{B}|f|^{2} d x d y$ is finite, in which case the integral equals $\|f\|^{\prime 2}$. Davis proved that a necessary and sufficient condition that (32) converged uniformly in $L^{\prime 2}(B)$ is that $\lim _{n \rightarrow \infty}\left\|E_{n}\right\|^{\prime 2}=0$. A similar result can be shown to hold for $L^{2}(B)$, the set of functions analytic within $B$ such that $\int_{B}|f(z)|^{2}\left|1-z^{2}\right|^{-1 / 2}|d z|$ is finite, where $\|f\|^{2}$ is defined accordingly.

Davis then showed that a necessary and sufficient condition that (32) converge uniformly in $L^{\prime 2}\left(\epsilon_{\rho}\right)$ is that

$$
\lim _{n \rightarrow \infty} \frac{4}{\pi} \sum_{k=0}^{\infty}(k+1) \frac{\left|E_{n}\left(U_{k}\right)\right|^{2}}{\rho^{2 k+2}-\rho^{-2 \bar{k}-\overline{2}}}=0 .
$$

A similar result holds for $L^{2}\left(\epsilon_{\rho}\right)$, with (33) replaced by

$$
\lim _{n \rightarrow \infty} \frac{2}{\pi} \sum_{k=0}^{\infty} \frac{\left|E_{n}\left(T_{k}\right)\right|^{2}}{\rho^{2 k}+\rho^{-2 k}}=0
$$

Let us now define an interpolatory quadrature scheme as one for which $E_{n}(f)=0$ if $f$ is a polynomial of degree $\leqq n$, and let us set $M_{n}=\sum_{k=0}^{n}\left|w_{k n}\right| \geqq 2$. Then Davis showed that a sufficient condition that an interpolatory quadrature scheme converge uniformly in $L^{\prime 2}\left(\epsilon_{\rho}\right)$ is that $\lim _{n \rightarrow \infty} M_{n} n^{3 / 2} \rho^{-n}=0$. For $L^{2}\left(\epsilon_{\rho}\right)$, the condition is slightly weaker, as given in the following theorem.

THEOREM. Let $\lim _{n \rightarrow \infty} M_{n} \rho^{-n}=0$. Then the interpolatory quadrature scheme converges uniformly in $L^{2}\left(\epsilon_{\rho}\right)$. 


\begin{tabular}{|c|c|c|c|}
\hline$a$ & $\rho$ & $\tau^{*}(\rho, T)$ & $\tau^{*}(\rho, S)$ \\
\hline 1.01 & 1.152 & $1.08(1)$ & \\
\hline 1.02 & 1.221 & $4.56(0)$ & \\
\hline 1.03 & 1.277 & 2.75 & \\
\hline 1.04 & 1.326 & 1.93 & \\
\hline 1.05 & 1.370 & 1.46 & $1.83(2)$ \\
\hline 1.06 & 1.412 & 1.17 & 1.02 \\
\hline 1.07 & 1.451 & $9.63(-1)$ & $6.17(1)$ \\
\hline 1.08 & 1.488 & 8.17 & 4.01 \\
\hline 1.09 & 1.524 & 7.07 & 2.75 \\
\hline 1.10 & 1.558 & 6.21 & 1.96 \\
\hline 1.12 & 1.624 & 4.97 & 1.09 \\
\hline 1.14 & 1.687 & 4.12 & $6.65(0)$ \\
\hline 1.16 & 1.748 & 3.51 & 4.34 \\
\hline 1.18 & 1.806 & 3.04 & 2.98 \\
\hline 1.20 & 1.863 & 2.68 & 2.13 \\
\hline 1.25 & 2.000 & 2.05 & 1.05 \\
\hline 1.30 & 2.131 & 1.66 & $5.89(-1)$ \\
\hline 1.35 & 2.257 & 1.38 & 3.63 \\
\hline 1.40 & 2.380 & 1.18 & 2.39 \\
\hline 1.45 & 2.500 & 1.03 & 1.65 \\
\hline 1.50 & 2.618 & $9.11(-2)$ & 1.19 \\
\hline 1.60 & 2.849 & 7.36 & $6.80(-2)$ \\
\hline 1.70 & 3.075 & 6.14 & 4.24 \\
\hline 1.80 & 3.297 & 5.23 & 2.83 \\
\hline 1.90 & 3.516 & 4.53 & 1.99 \\
\hline 2.00 & 3.732 & 3.98 & 1.45 \\
\hline 2.20 & 4.160 & 3.16 & $8.45(-3)$ \\
\hline 2.40 & 4.582 & 2.58 & 5.37 \\
\hline 2.60 & 5.000 & 2.16 & 3.63 \\
\hline 2.80 & 5.415 & 1.83 & 2.57 \\
\hline 3.00 & 5.828 & 1.58 & 1.89 \\
\hline 4.00 & 7.873 & $8.60(-3)$ & $5.75(-4)$ \\
\hline 5.00 & 9.900 & 5.43 & 2.54 \\
\hline 6.00 & 11.920 & 3.75 & 1.41 \\
\hline 7.00 & 13.928 & 2.74 & $9.02(-5)$ \\
\hline 8.00 & 15.937 & 2.09 & 6.38 \\
\hline 9.00 & 17.944 & 1.65 & 4.80 \\
\hline 10.00 & 19.950 & 1.34 & 3.78 \\
\hline 20.00 & 39.975 & $3.33(-4)$ & $8.91(-6)$ \\
\hline
\end{tabular}

TABLE 1. Values of $\tau^{*}(\rho, R)$ for various values of $\rho$ and for the trapezoidal $(T)$ and Simpson $(S)$ rules. 
Proof. We have

$$
\left\|E_{n}\right\|^{2}=C_{1} \sum_{k=n+1}^{\infty} \frac{\left[\frac{1+(-1)^{k}}{1-k^{2}}-\sum_{j=0}^{n} w_{n i} T_{k}\left(x_{n j}\right)\right]^{2}}{\rho^{2 k}+\rho^{-2 k}} .
$$

Since $\rho^{2 k} \leqq \rho^{2 k}+\rho^{-2 k} \leqq 2 \rho^{2 k}$, we have

$$
\begin{aligned}
C_{2}\left\|E_{n}\right\|^{2} & \leqq \sum_{k=n+1}^{\infty} \frac{\left[\frac{2}{k^{2}-1}+\sum_{i=0}^{n}\left|w_{n i}\right|\left|T_{k}\left(x_{n j}\right)\right|\right]^{2}}{\rho^{2 k}} \\
& \leqq \sum_{k=n+1}^{\infty}\left(\frac{2}{k^{2}-1}+M_{n}\right)^{2} \rho^{-2 k} \leqq\left[\frac{4}{n^{4}}+\frac{4 M_{n}}{n^{2}}+M_{n}^{2}\right] \sum_{k=n+1}^{\infty} \rho^{-2 k} \\
& =\left[\frac{4}{n^{4}}+\frac{4 M_{n}}{n^{2}}+M_{n}^{2}\right] \frac{\rho^{-2 n}}{\rho^{2}-1}=C_{3} \rho^{-2 n}+C_{4} M_{n} n^{-2} \rho^{-2 n}+C_{5} M_{n}^{2} \rho^{-2 n}
\end{aligned}
$$

Therefore, $\lim _{n \rightarrow \infty} M_{n} \rho^{-n}=0$ implies $\lim _{n \rightarrow \infty}\left\|E_{n}\right\|^{2}=0$. For Newton-Cotes integration rules,

$$
M_{n} \leqq \frac{4\left(1+\delta_{n}\right) 2^{n}}{n(\log n)^{2}}+\frac{4\left(1+\epsilon_{n}\right)}{n \log n},
$$

where $\delta_{n}, \epsilon_{n} \rightarrow 0$ as $n \rightarrow \infty$. The condition of the above theorem then holds for $\rho \geqq 2$. Therefore, the Newton-Cotes quadrature scheme converges uniformly in $L^{2}\left(\epsilon_{\rho}\right)$ whenever $\rho \geqq 2$. Davis' analogous result for $L^{\prime 2}\left(\epsilon_{\rho}\right)$ holds with $\rho>2$.

Department of Applied Mathematics

Weizmann Institute of Science

Rehovot, Israel

1. R. E. BARNHILl \& J. A. WIXOM, "Quadratures with remainders of minimum norm. I, II," Math. Comp., v. 21, 1967, pp. 66-75, 382-387. MR 36 \#6138; MR 36 \#6139.

2. M. M. CHAWLA, "Asymptotic estimates for the error of the Gauss-Legendre quadrature formula," Comput. J., v. 11, 1968/69, pp. 339-340. MR 38 \#5389.

3. P. J. DAvis, "Errors of numerical approximation for analytic functions," J. Rational Mech. Anal., v. 2, 1953, pp. 303-313. MR 14, 907.

4. P. J. Davis, Errors of Numerical Approximation for Analytic Functions, Survey of Numerical Analysis, McGraw-Hill, New York, 1962, pp. 468-484. MR 24 \#B1766.

5. P. J. DAvis, Interpolation and Approximation, Blaisdell, Waltham, Mass., 1963. MR 28 \#393.

6. P. J. Davis \& P. Rabinowitz, "On the estimation of quadrature errors for analytic functions," $M T A C$, v. 8, 1954, pp. 193-203. MR 16, 404.

7. G. HäMMERLIN, "Zur Abschätzung von Quadraturfehlern für analytische Funktionen," Numer. Math., v. 8, 1966, pp. 334-344. MR 34 \#2179.

8. D. Nicholson, P. Rabinowitz, N. Richter \& D. Zeilberger, "On the Error in the Numerical Integration of Chebyshev Polynomials," Math. Comp., v. 25, 1971.

9. P. RABINOWITZ, "Practical error coefficients for estimating quadrature errors for analytic functions," Comm. ACM, v. 11, 1968, pp. 45-46, MR 39 \#2324.

10. P. RABINOWITZ \& N. RichTER, "Asymptotic properties of minimal integration rules," Math. Comp., v. 24, 1970, pp. 593-609.

11. A. H. STRoud \& D. SECREST, Gaussian Quadrature Formulas, Prentice-Hall, Englewood Cliffs, N. J., 1966. MR 34 \#2185. 